\title{
Anti-Icing Property of Superhydrophobic Octadecyltrichlorosilane Film and Its Ice Adhesion Strength
}

\author{
Liang Ge, Guifu Ding, Hong Wang, Jinyuan Yao, Ping Cheng, and Yan Wang \\ Science and Technology on Micro/Nano Fabrication Laboratory, Research Institute of Micro/Nano Science and Technology, \\ Shanghai Jiao Tong University, Shanghai 200240, China
}

Correspondence should be addressed to Yan Wang; wyyw@sjtu.edu.cn

Received 23 January 2013; Accepted 30 March 2013

Academic Editor: Huijun Wu

Copyright (C) 2013 Liang Ge et al. This is an open access article distributed under the Creative Commons Attribution License, which permits unrestricted use, distribution, and reproduction in any medium, provided the original work is properly cited.

An octadecyltrichlorosilane (OTS) superhydrophobic film using phase-separation method was prepared to demonstrate the antiicing property of superhydrophobic surfaces. The superhydrophobicity of the film at $-5^{\circ} \mathrm{C}$ was investigated. It was found that the prepared OTS film retained its superhydrophobicity at $-5^{\circ} \mathrm{C}$ by the measurement of contact angle and roll-off angle. The icing progress of water droplets on the surface at $-15^{\circ} \mathrm{C}$ was observed. It showed that the prepared OTS film can markedly retard the icing process of water droplets and dramatically decrease the ice adhesion strength compared with that of blank surface, which can be used as anti-icing surfaces.

\section{Introduction}

It is well known that icing occurs when water changes from liquid to solid phase. Ice adhesion and accumulation on critical parts of structures will cause damaging problems, severe accidents, and economic losses in the fields of aerospace, transportation and power communication. For instance, droplets of supercooled water often exist in stratiform and cumulus clouds. Aircraft flying through these clouds seed an abrupt crystallization of these droplets, which can result in the formation of ice on the aircraft's wings or blockage of its instruments, unless the aircrafts are equipped with an appropriate deicing system. Icing on plane will reduce the handling and stability performance, which may cause aviation accident.

Superhydrophobicity has drawn widespread attention for its self-cleaning character and potential application in fluid devices, water repellency textile and functional separation equipment [1-4]. Generally, a surface with a contact angle larger than $150^{\circ}$ and a roll-off angle less than $10^{\circ}$ is defined as superhydrophobic surface. A few kinds of superhydrophobic surfaces have been developed by self-assembling [5-8], etching $[9,10]$, phase-separation method $[1,11]$, and so forth.
Numerous researchers have reported the application of superhydrophobic surface on water repellency at room temperature $[12,13]$. It has been reported that icing may be retarded on superhydrophobic surface [14]. However, few researches were concerned with the possibility of applying superhydrophobic surface in subzero environment. And the effect of surface wettability on retarding ice formation still needs experimental investigation.

In this paper, a facile method to prepare superhydrophobic surface by phase-separation technology was proposed. The hydrophobicity of the prepared OTS film was greatly enhanced compared to the conventional dip-dry method with the contact angle less than $120^{\circ}$ [10]. To carry out the icing experiment, a small cold storage was established which could offer temperature low at $-30^{\circ} \mathrm{C}$. Both of the static and dynamic hydrophobicities of the prepared surface were studied in subzero environment. The icing process on the superhydrophobic surface was also observed. Based on the classical heterogeneous nucleation theory, the icing behaviors of droplets on superhydrophobic surface were discussed. Based on the bonding forces measured between the prepared surface and ice, the ice adhesion strengths were calculated and compared with those of blank surface. 


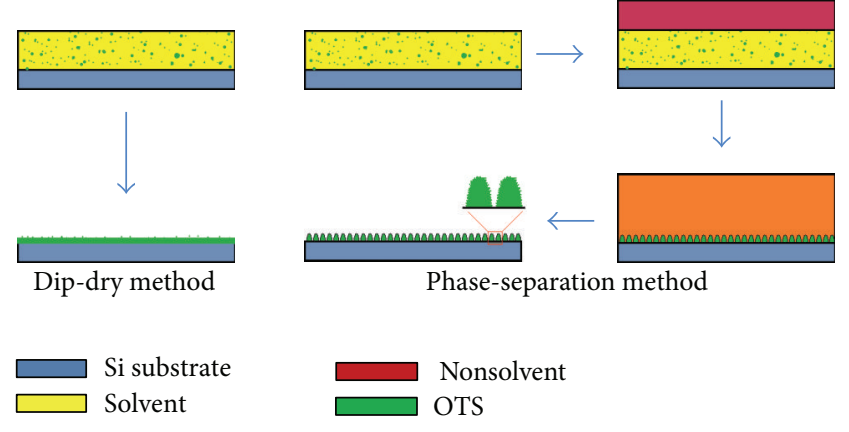

Figure 1: Comparison of one-step dip-dry method and phaseseparation method.

\section{Materials and Methods}

2.1. Preparation of Samples. The substrate used in this study is silicon wafer with a radius of 3 inches and a thickness of $0.45 \mathrm{~mm}$. A coating solution of $0.1 \mathrm{~mL}$ octadecyltrichlorosilane (OTS, Acros Organics, USA) that dissolved in $30 \mathrm{~mL}$ normal hexane was prepared. Dichloromethane and acetone were selected as nonsolvents. The clean silicon wafer was dipped into the coating solution for 1 minute. After evaporation for 1 minute at room temperature, the substrate coated with OTS film was immersed in the nonsolvent for 5 minutes, where a phase-separation process took place and the micronano rough surface structure formed. Figure 1 illustrates the procedures of one-step dip-dry method and phase-separation process.

In a phase-separation progress, it is of common occurrence that solid/liquid phases separate and liquid/liquid phases separate. Due to the interactions of these phaseseparation processes, the solute will form rough microstructures after crystallization. The rough microstructures usually possess nanometer scale roughness, while the microstructures formed using the conventional dip-dry method can only show micrometer scale roughness.

2.2. Characterization and Test. The micrographs of the OTS film were characterized by a scanning electron microscope (Zeiss Ultra55). A video-based contact angle measurement system (POWEREACH, JC2000D) was used to measure and record the water contact angles (WCAs) and roll-off angles of the samples. The static WCAs were measured at least 3 times using the sessile drop method by dispersing $5 \mu \mathrm{L}$ drops of deionized water onto the sample surface. The roll-off angles were measured by tilting the substrate and the slant angles were recorded when the droplet rolled off across the surface in a downhill direction. A small cold storage was established, where the temperature could be adjusted between $0^{\circ} \mathrm{C}$ and $-30^{\circ} \mathrm{C}$ by a refrigerating unit. To measure the contact and roll-off angles in subzero environment, the temperature in the cold store was set at $-5^{\circ} \mathrm{C}$. The relative humidity was $40 \%$. The water and the substrate were cooled at $-5^{\circ} \mathrm{C}$ for $3 \mathrm{~h}$ before the test.

The icing process was performed in the cold storage from $-9^{\circ} \mathrm{C}$ to $-15^{\circ} \mathrm{C}$. The droplets were dropped onto the treated

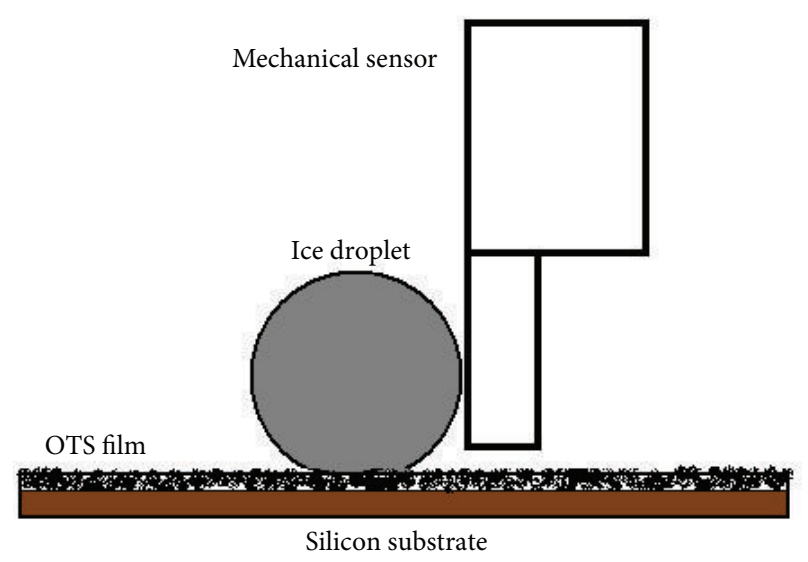

Figure 2: Schematic diagram of the bonding force test.

superhydrophobic surface by $1 \mathrm{~mL}$ syringe at $1^{\circ} \mathrm{C}$. Then the temperature was reduced until icing process finished. The status of the droplets was recorded by digital camera every hour.

The bonding force between ice droplets and the film surface was tested at $-15^{\circ} \mathrm{C}$ using a bonding force measurement system (RHESCA, PTR-1101). Figure 2 illustrated schematic diagram of the bonding force test.

As depicted in Figure 2, the water droplet froze on the OTS film surface coated on the silicon substrate which was fixed on the objective table. The probe with a mechanical sensor was set at an appropriate position by the controller. Then the probe began to move horizontally at $1 \mathrm{~mm} / \mathrm{s}$ and push the ice droplet. The instantaneous value of the shear force was recorded by the mechanical sensor. The contact area between the surface and ice droplet was calculated based on the image of the ice droplet taken just before the bonding force test. The shear stress was then calculated as the ratio of the shear force and the contact area. The ice adhesion strength was the shear stress at which the ice droplet detached from the sample surface as indicated by the sudden drop of the sensed shear force to zero.

\section{Results and Discussion}

3.1. Surface Topography and Wettability at $-5^{\circ} \mathrm{C}$. Figure 3 showed the SEM images of the OTS films surface with and without treatment of nonsolvents. It can be observed from Figures 3(b) and 3(c) that there are more protrusions and voids on the treated OTS film than that on the untreated OTS film. The treatment of the nonsolvent notably changed the topography of OTS film surface. The disordered reticular structure with a submicron/nanometer size on the surface played a crucial role in the superhydrophobic property. The static contact angles and roll-off angles of the various OTS films at $-5^{\circ} \mathrm{C}$ were listed in Table 1 .

As shown in Figure 3 and Table 1, the surface of OTS film without further treatment was hydrophobic with a contact angle of $140^{\circ}$ and a roll-off angle larger than $90^{\circ}$. Both the OTS films treated with dichloromethane and acetone 


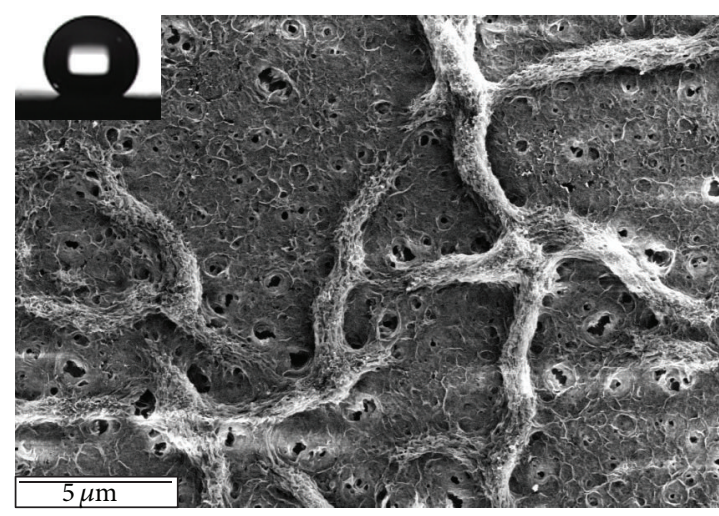

(a)

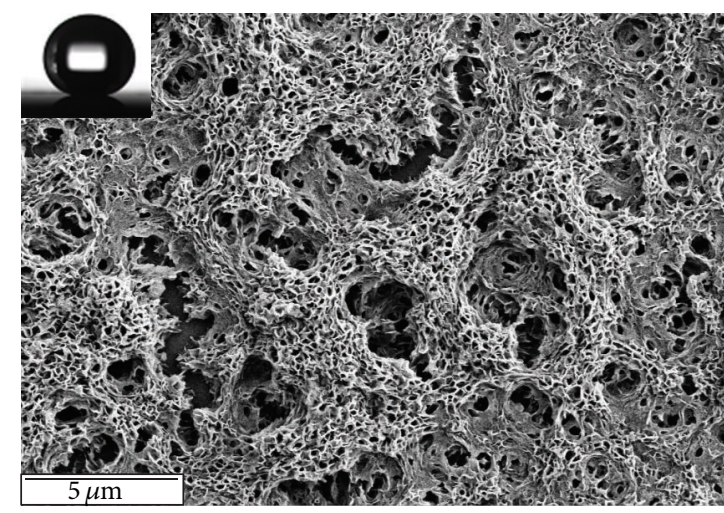

(b)

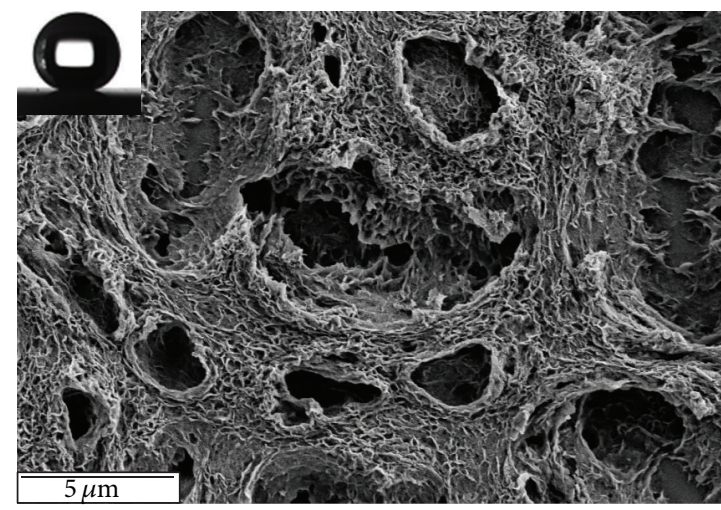

(c)

FIGURE 3: SEM images of various OTS films-(a) without treatment, (b) dichloromethane treatment, and (c) acetone treatment.

TABLE 1: Contact and roll-off angles of OTS films at $-5^{\circ} \mathrm{C}$ without and with nonsolvent treatment.

\begin{tabular}{lccc}
\hline Samples & Nonsolvent & WCA & Roll-off angle \\
\hline a & Without & $140^{\circ}$ & $\geq 90^{\circ}$ \\
b & Dichloromethane & $155^{\circ}$ & $4^{\circ}$ \\
c & Acetone & $155^{\circ}$ & $10^{\circ}$ \\
\hline
\end{tabular}

had the same contact angles of $155^{\circ}$ and roll-off angles less than $10^{\circ}$. Superhydrophobicity is determined mainly by the characteristics of the solid surface. This phenomenon can be explained by the Cassie-Baxter model [15]. Cassie and Baxter proposed a gas cavity model for porous surface wettability. According to the Cassie-Baxter model, air is more likely to be trapped between the water droplets and the protrusions of the porous surface. The following equation is given for the contact angle of droplet on a real surface:

$$
\cos \theta^{\prime}=f(1+\cos \theta)-1
$$

where $\theta^{\prime}$ is the contact angle for a real rough surface, $\theta$ is the contact angle for the corresponding ideal smooth surface, and $f(f<1)$ is the fraction of solid surface area wet by the liquid. From (1), the contact angle of a real surface is always larger than that of an ideal surface under the same conditions as $f<1$. In this work, as shown in Figures 3 and 4 ,

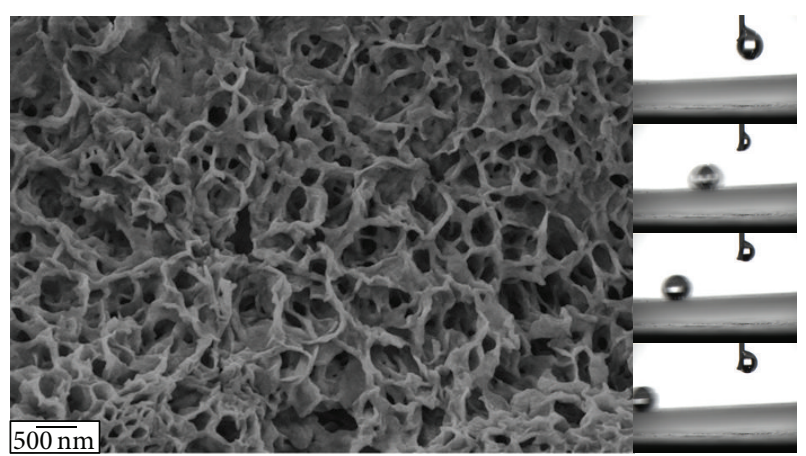

FIGURE 4: SEM image of OTS film treated with dichloromethane (left) and its dynamic hydrophobicity test (right) at $-5^{\circ} \mathrm{C}$.

there were many micro-nano scale protrusions on the coated silicon surface, trapping the air easily under the water droplet, so the contact fraction $f$ decreased and the WCA was much larger than that of a smooth surface. Thus, the coating film increased the hydrophobicity of the surface and provided a superb superhydrophobic surface.

The complicated rough micro-nano binary structures modulated the three-phase contact line from continuity to discrete, which resulted in smaller adhesion force and rolloff angle [16]. So, the protrusions and voids on the surface 


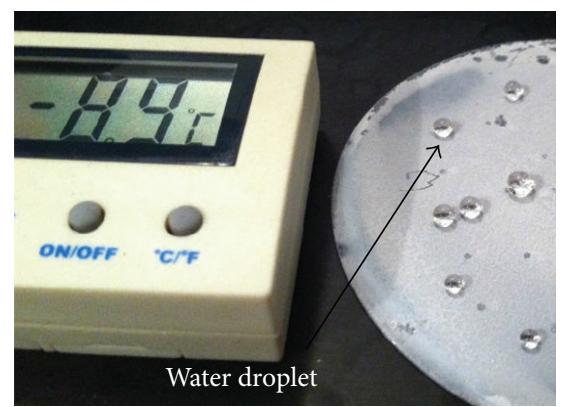

(a) $60 \mathrm{~min}$ at $-9^{\circ} \mathrm{C}$

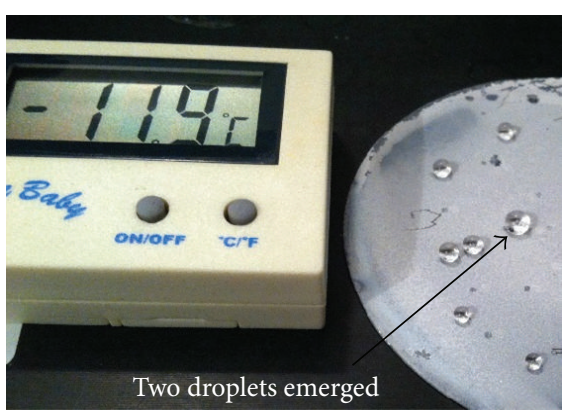

(b) 60 min at $-12^{\circ} \mathrm{C}$

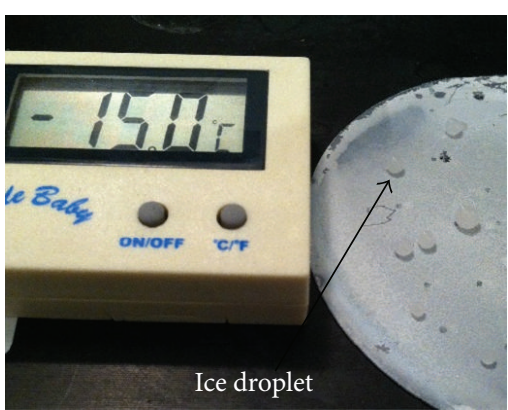

(c) 60 min at $-15^{\circ} \mathrm{C}$

FIGURE 5: Icing process of water droplets on the OTS film treated with dichloromethane.

contributed to the greater contact angles and smaller roll-off angles for the OTS films treated with dichloromethane and acetone. As the OTS film treated with dichloromethane had a smaller roll-off angle $\left(4^{\circ}\right)$ than that by acetone $\left(10^{\circ}\right)$, it was taken for an example to study the anti-icing property as well as ice adhesion strength in the following sections. Figure 4 showed the dynamic hydrophobicity of the OTS film treated with dichloromethane. The water droplet of $5 \mu \mathrm{L}$ rolled off the film with a tilting angle of $4^{\circ}$ in $0.2 \mathrm{~s}$. The images of the rolloff angle were recorded by CCD camera. It could be observed that the roll-off angle was $4^{\circ}$ and the contact angle was $155^{\circ}$.

3.2. Icing Behavior. Water was dropped onto OTS film treated with dichloromethane at $1^{\circ} \mathrm{C}$ and placed in the cold storage at $0^{\circ} \mathrm{C}$ for $3 \mathrm{~h}$. The icing process was recorded by digital camera, as shown in Figure 5.

As shown in Figure 5, the water droplets remained in liquid state at a temperature low to $-12^{\circ} \mathrm{C}$ on the prepared surface and did not freeze until the temperature was reduced to $-15^{\circ} \mathrm{C}$. The water droplets in Figures 5(a) and 5(b) could be distinguished from the ice droplets in Figure 5(c) by the reflection of light. The experimental results indicated that the icing process of water droplets on prepared superhydrophobic surface was greatly retarded, which demonstrated the superb anti-icing property of the film.

This phenomenon could be explained by the heterogeneous nucleation theory $[14,17]$. It is well known that several factors, including critical nucleation radius, nucleation radius, temperature, humidity, pressure, and surface condition, contributed to the icing process of water droplet on solid surface. However, the surface condition is the dominant factor that determines the icing process under the same conditions. Compared to homogeneous nucleation theory, the free energy barrier of heterogeneous nucleation on the surface could be expressed as a function of the contact angle $\theta$ between droplets and the surface, which monotonously increased with the contact angle $\theta[14,17]$.

Because the icing probability was dependent on the free energy barrier (the lower the free energy barrier is, the easier the droplet freezes), the phenomenon that the droplet on hydrophilic surface was more incidental to freeze than that on hydrophobic surface could be explained. In this study,

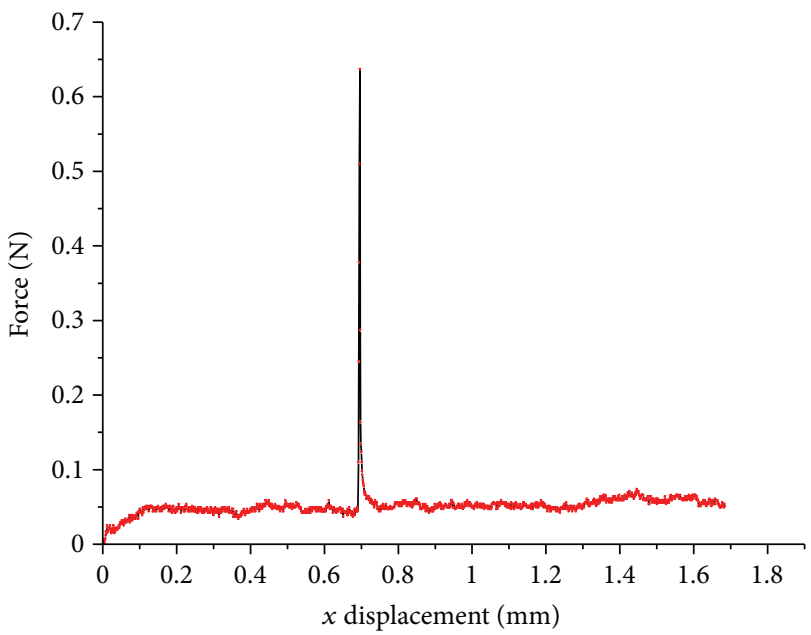

FIGURE 6: Displacement-force curve during ice adhesion test.

the water contact angle of the OTS superhydrophobic surface was $155^{\circ}$, while the contact angle for clean silicon surface is seldom less than $50^{\circ}$ (see Figure 7). Thus, the potential barrier of OTS superhydrophobic surface was much larger than that of the clean silicon surface. Accordingly, the critical nucleation radius was bigger for the superhydrophobic surface. Consequently, the OTS superhydrophobic surface can retard the droplet icing on it, and the time needed to ice on OTS superhydrophobic surface is much longer than that on clean silicon surface. These results demonstrate the significant applicable probability of superhydrophobic surface in preventing the ice formation.

3.3. Ice Adhesion Strength. In order to investigate the ice adhesion strength on the superhydrophobic surface, the shear forces of ice droplets formed on the samples were measured using a bonding force measurement system. Figure 6 showed a displacement-force curve during ice bonding force test of OTS film surface. The sudden drop of shear force to zero indicates the ice detachment. Same tests were conducted at 20 different points on OTS film surface and 8 different points on clean silicon wafer. 


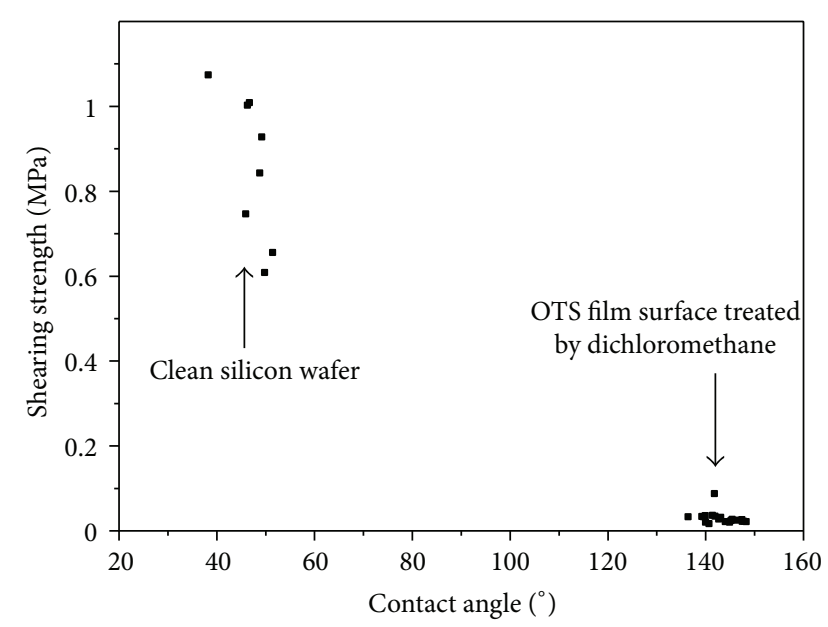

FIGURE 7: Dependence of the ice adhesion shear strength on contact angle.

Figure 7 compared the ice adhesion strength on clean silicon wafer and that on OTS film treated with dichloromethane. It showed that the ice adhesion strength on superhydrophobic surface averagely decreases $95.74 \%$ more than that on clean silicon wafer, which clearly demonstrates the outstanding performance of the superhydrophobic film on deicing. It was suggested that the disordered reticular structure plays a crucial role in the low adhesion strength between ice droplet and OTS superhydrophobic surface. In addition, during the shear forces test, the ice droplets were detached without exfoliation of the OTS film. This indicates that the adhesion strength of the OTS film on the silicon wafer is larger than that of the ice droplets on the coating film.

\section{Conclusion}

In this research, a superhydrophobic surface was prepared, using a simple phase-separation method. The results showed that the surface retained its excellent superhydrophobicity even in subzero environment. The superhydrophobic property was attributed to the disordered reticular structure in submicron/nanometer scale caused by the phase-separation method. The superhydrophobic surface also showed excellent anti-icing property. It was found that the OTS film surface treated with dichloromethane could markedly retard the icing progress of water droplets, as well as dramatically decrease the adhesion strength between ice droplets and the surface compared with the clean silicon surface. This indicates that the prepared OTS film surface in the study had an outstanding anti-icing performance.

\section{Acknowledgments}

The research is supported by Shanghai Science and Technology Development Foundation (no. 11DZ2290203) and the National Key Laboratory Foundation (9140C790103120C7906).

\section{References}

[1] H. Y. Erbil, A. L. Demirel, Y. Avci, and O. Mert, “Transformation of a simple plastic into a superhydrophobic surface," Science, vol. 299, no. 5611, pp. 1377-1380, 2003.

[2] T. Sun, L. Feng, X. Gao, and L. Jiang, "Bioinspired surfaces with special wettability," Accounts of Chemical Research, vol. 38, no. 8, pp. 644-652, 2005.

[3] M. Ma and R. M. Hill, "Superhydrophobic surfaces," Current Opinion in Colloid and Interface Science, vol. 11, no. 4, pp. 193202, 2006.

[4] L. Feng, Z. Yang, J. Zhai et al., "Superhydrophobicity of nanostructured carbon films in a wide range of $\mathrm{pH}$ values," Angewandte Chemie International Edition, vol. 42, no. 35, pp. 4217-4220, 2003.

[5] M. E. McGovern, K. M. R. Kallury, and M. Thompson, "Role of solvent on the silanization of glass with octadecyltrichlorosilane," Langmuir, vol. 10, no. 10, pp. 3607-3614, 1994.

[6] A. Ulman, "Formation and structure of self-assembled monolayers," Chemical Reviews, vol. 96, no. 4, pp. 1533-1554, 1996.

[7] K. C. Grabar, P. C. Smith, M. D. Musick et al., "Kinetic control of interparticle spacing in Au colloid-based surfaces: rational nanometer-scale architecture," Journal of the American Chemical Society, vol. 118, no. 5, pp. 1148-1153, 1996.

[8] D. I. Gittins, D. Bethell, R. J. Nichols, and D. J. Schiffrin, "Redox-connected multilayers of discrete gold particles: a novel electroactive nanomaterial," Advanced Materials, vol. 11, no. 9, pp. 737-740, 1999.

[9] J. Wang, F. Liu, H. Chen, and D. Chen, "Superhydrophobic behavior achieved from hydrophilic surfaces," Applied Physics Letters, vol. 95, no. 8, Article ID 084104, 2009.

[10] L. Zhu, Y. Feng, X. Ye, and Z. Zhou, “Tuning wettability and getting superhydrophobic surface by controlling surface roughness with well-designed microstructures," Sensors and Actuators A, vol. 130-131, pp. 595-600, 2006.

[11] C. Y. Kuo, H. N. Lin, H. A. Tsai, D. M. Wang, and J. Y. Lai, "Fabrication of a high hydrophobic PVDF membrane via nonsolvent induced phase separation," Desalination, vol. 233, no. 1-3, pp. 40-47, 2008.

[12] X. Song, J. Zhai, Y. Wang, and L. Jiang, "Fabrication of superhydrophobic surfaces by self-assembly and their wateradhesion properties," Journal of Physical Chemistry B, vol. 109, no. 9, pp. 4048-4052, 2005.

[13] T. Hang, A. Hu, H. Ling, M. Li, and D. Mao, "Superhydrophobic nickel films with micro-nano hierarchical structure prepared by electrodeposition," Applied Surface Science, vol. 256, no. 8, pp. 2400-2404, 2010.

[14] L. Cao, A. K. Jones, V. K. Sikka, J. Wu, and D. Gao, "AntiIcing superhydrophobic coatings," Langmuir, vol. 25, no. 21, pp. 12444-12448, 2009.

[15] A. B. D. Cassie and S. Baxter, "Wettability of porous surfaces," Transactions of the Faraday Society, vol. 40, pp. 546-551, 1944.

[16] Y. Lai, X. Gao, H. Zhuang, J. Huang, C. Lin, and L. Jiang, "Designing superhydrophobic porous nanostructures with tunable water adhesion," Advanced Materials, vol. 21, no. 37, pp. 3723-3803, 2009.

[17] X. Y. Liu, "A new kinetic model for three-dimensional heterogeneous nucleation," Journal of Chemical Physics, vol. 111, no. 4, pp. $1628-1635,1999$. 

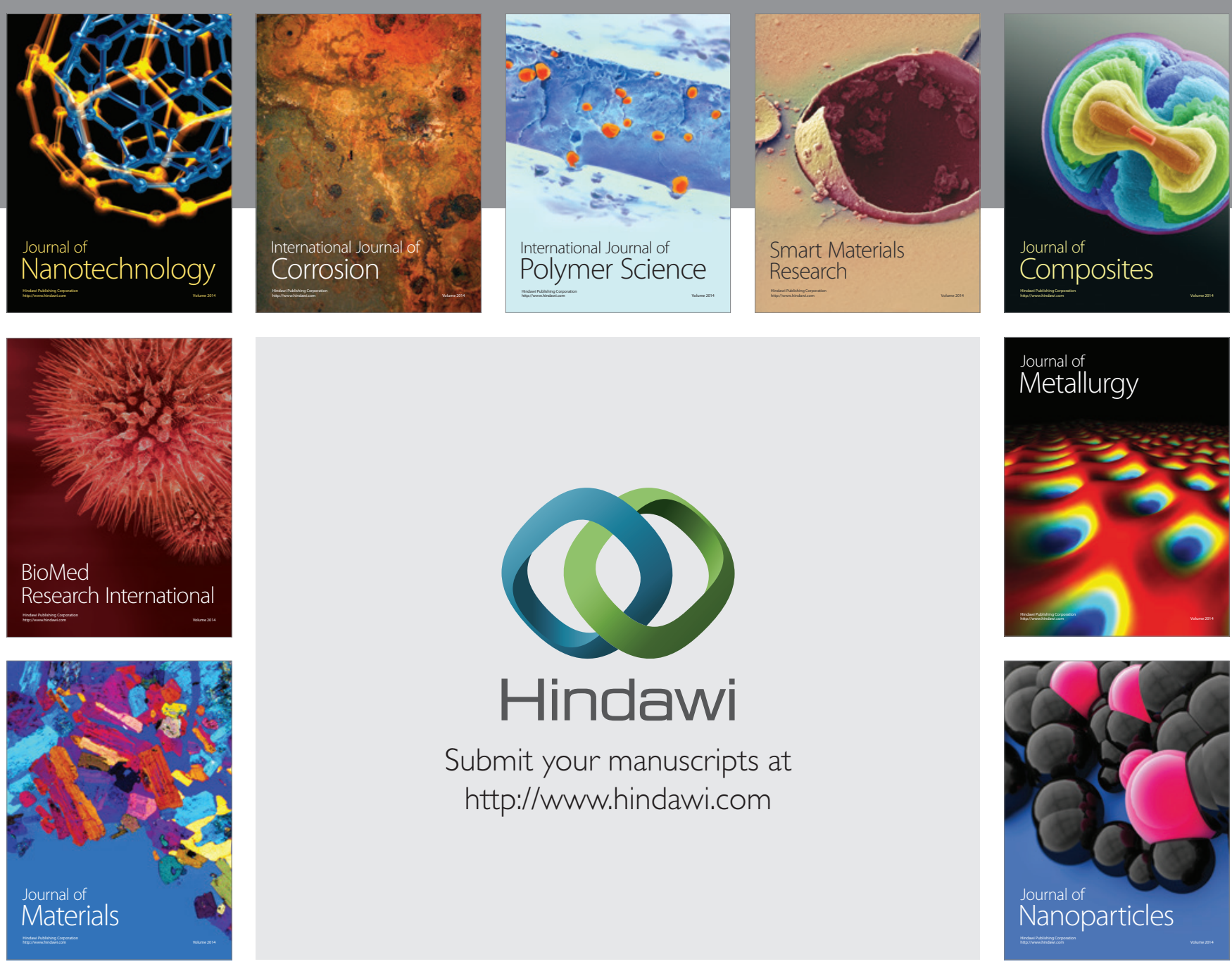

Submit your manuscripts at http://www.hindawi.com
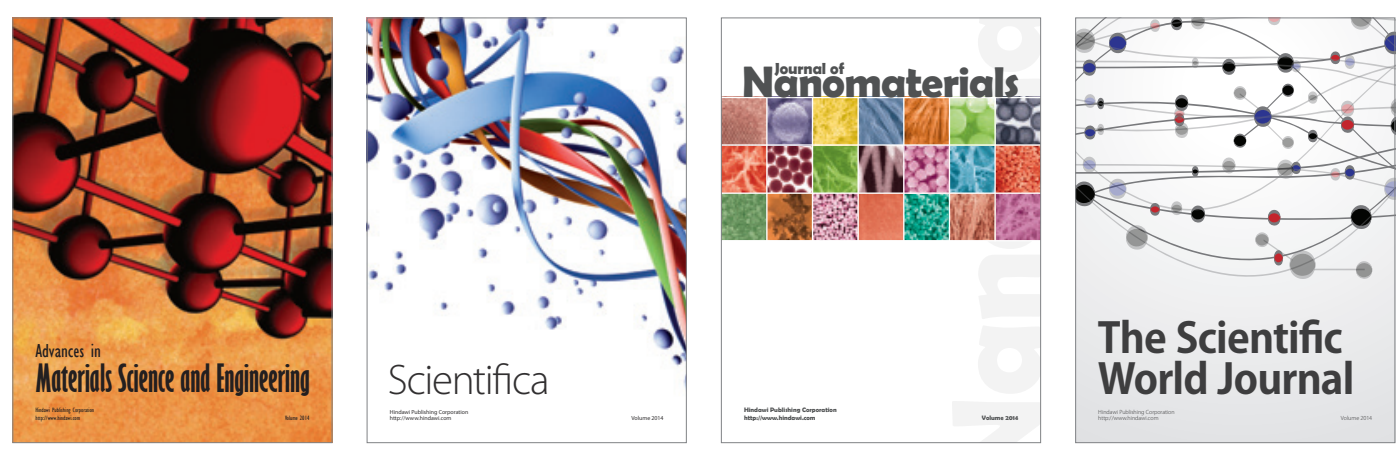

\section{The Scientific World Journal}
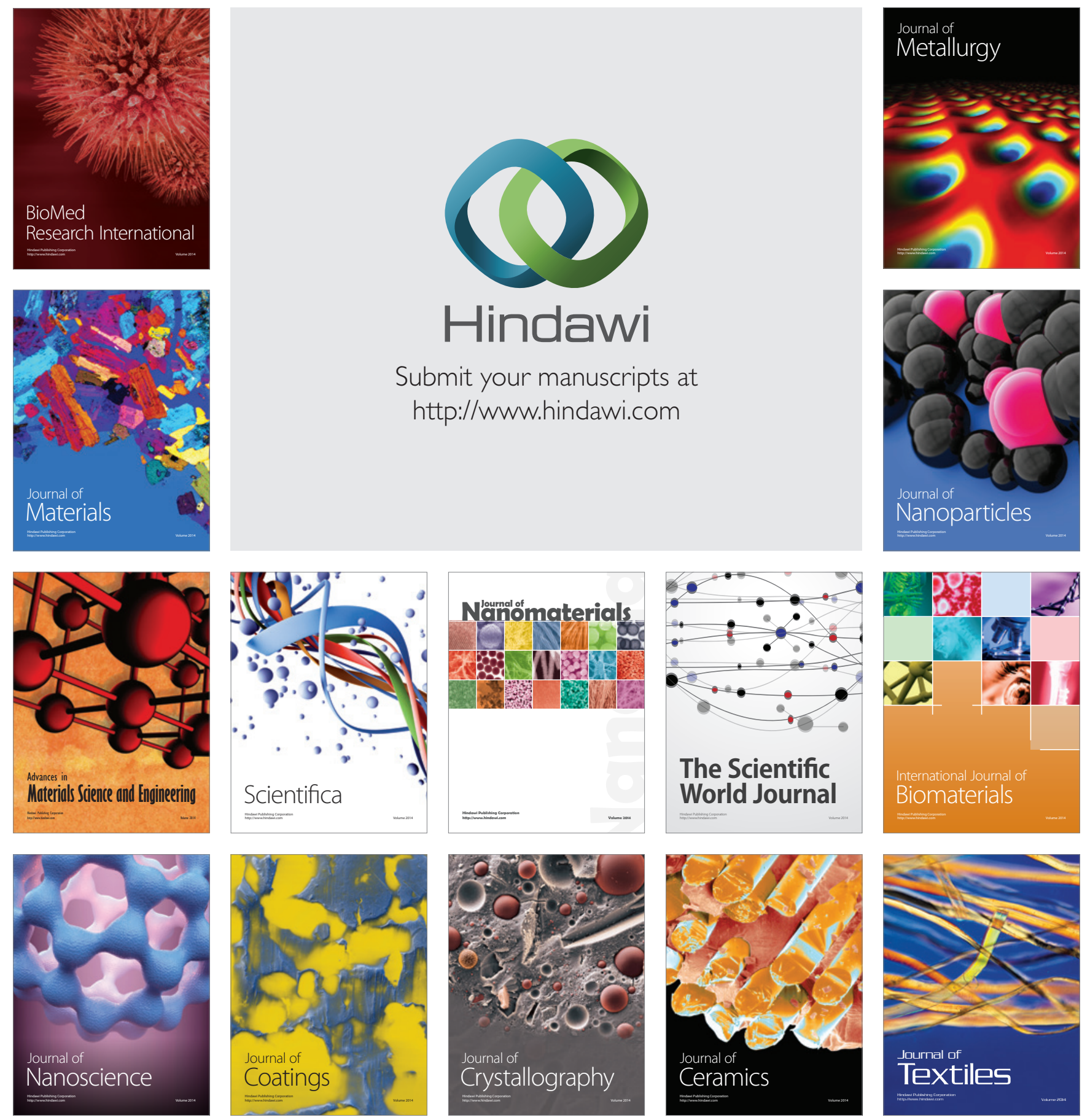